\title{
An artificial neural network application on nuclear charge radii
}

\author{
Serkan Akkoyun, Tuncay Bayram, S. Okan Kara and Alper Sinan \\ Department of Physics, Cumhuriyet University, Sivas, Turkey \\ Department of Physics, Sinop University, 57000 Sinop, Turkey \\ Department of Physics, Nigde University, Nigde, Turkey \\ Department of Statistics, Sinop University, 57000 Sinop, Turkey
}

\begin{abstract}
The artificial neural networks (ANNs) have emerged with successful applications in nuclear physics as well as in many fields of science in recent years. In this paper, by using (ANNs), we have constructed a formula for the nuclear charge radii. Statistical modeling of nuclear charge radii by using ANNs has been seen as to be successful. Also, the charge radii, binding energies and two-neutron separation energies of Sn isotopes have been calculated by implementing of the new formula in Hartree-Fock-Bogoliubov (HFB) calculations. The results of the study shows that the new formula is useful for describing nuclear charge radii.
\end{abstract}

Keywords: Nuclear charge radii, Artificial neural network, HartreeFock-Bogoliubov method

\section{Introduction}

Nuclear charge radii and, more generally, distributions of charge-density give direct information on the Coulomb energy of nuclei. Because of this reason, it has gained attention for the nuclear mass formulas in recent six decades [1]. As is well known, the nuclear charge radii is a fundamental property of atomic nuclei 213. It can be measured by various methods based on the electromagnetic interaction between the nucleus and electrons or muons. Widely used methods are measurements of transition energies in muonic atoms, elastic electron scattering experiments, $K_{\alpha} \mathrm{X}$-ray and optical isotope shifts. A detailed discussions on these techniques for measuring of the rms (root-mean-square) charge radii of nuclei can be found in [4. The latest advances in experimental techniques have provided accessibility to experimental nuclear charge of more nuclei far from $\beta$-stability line.

The nuclear charge radii related with exotic phenomena such as skin and halo has become a hot topic [5]. With its accuracy, the study of the nuclear charge radii is very important for a better understanding of not only the proton distribution in nuclei but also the skin and halo. For these reasons, if one get a simple and reliable formula for nuclear charge radii, it can be provides information for exotic nuclei and the effective nucleon-nucleon interaction. For these purpose, a 
number of nuclear charge formulae have been proposed as to be based on $A^{1 / 3}$ and $Z^{1 / 3}$ dependence. An excellent comprehensive study on nuclear charge formula obtained from fitting of experimental data can be found in [5] and reference therein. However, in these studies experimental data were limited. New experimental data over more than 800 nuclei have been updated, recently [4]. Using the new data, the parameters of the nuclear charge formulae can be revised. In the present work, we used feed-forward artificial neural networks (ANNs) as a different approach for obtaining a reliable and simple nuclear charge formula. As is well known, feed-forward ANNs may be viewed as an universal non-linear function approximator [6]. In recent years, ANNs have been used in many fields in nuclear physics as in the other fields, such as determination of one and two proton separation energies [7, developing nuclear mass systematics [8, identification of impact parameter in heavy-ion collisions [9|10|11, estimating beta decay half-lives [12] and obtaining potential energy curves [13]. The fundamental task of the ANNs is to give outputs in consequence of the computation of the inputs. We have obtained new mass dependent simple nuclear charge formula by using all the data from ANN whose inputs were taken from the latest experimental data. The obtained new formula is presented and used in HFB calculations for Sn nuclei.

The paper is organised as follows. In Section 2, ANNs formalism and HartreeFock-Bogoliubov (HFB) method are given briefly. In Section 3, the results of the study and discussions are presented. Finally, a summary is given in Section 4.

\section{Theoretical Framework}

\section{$2.1 \quad$ ANNs}

ANNs are mathematical models that mimic the human brain. They consist of several neurons which are processing units. The neurons are connected each other via adaptive synaptic weights [14]. The ANN is composed of three main layers. The first layer corresponds to input layer, the intermediate layer is called hidden layer and the last one is output layer. In this study, one input layer with a neuron, one hidden layer with forty four neurons $(\mathrm{h}=44)$ and one output layer with a neuron ANN was used. The total adjustable weights was $2 \mathrm{~h}=88$.

The input layer neuron receives the data from outside and the output layer neuron gives the results. The data is transmitted via weighted connections between the neurons. The tangent hyperbolic (sigmoidlike) and linear functions were used for hidden and output units respectively. It has been proven that one hidden layer and sigmoidlike activation function in this layer are sufficient to approximate any continuous real function [15].

The use of ANN is as two-step process, training and test stages. In this work, a back-propagation algorithm with Levenberg-Marquardt [16 17] for the training of the ANN was used. In the training stage, ANN modifies its weights until an acceptable error level between desired and predicted outputs is attained. The error function which measures this difference was mean square error (MSE). 
After an acceptable error level, the trained ANN is tested over the data of interest. For further and general background for the ANN, the reader is referred to [14].

\section{$2.2 \quad$ HFB Method}

In the HFB formalism, a two-body Hamiltonian of a system of fermions can be interpreted in terms of a set of annihilation and creation operators. The ground-state wave function is described as the quasi-particle vacuum and the linear Bogoliubov transformation provides connection between the quasiparticle operators and the original particle operators. The basic building blocks of the HFB method are the density matrix and the pairing tensor, and expectation value of the HFB Hamiltonian could be expressed as an energy functional (Details can be found in [218]). In terms of Skyrme forces, the HFB energy has the form of local energy density functional contains the sum of the mean field and pairing energy densities. These fields can be calculated in the coordinate space [2]8. In this work, HFB equations have been solved by expanding quasiparticle wave functions that conserve axial symmetry and parity on a harmonic oscillator basis expressed in coordinate space proposed by Stoitsov et al. 18. For pairing, Lipkin-Nogami method is implemented by performing the HFB calculations with an additional term included in the HF Hamiltonian. To obtain ground-state properties of Sn isotopes prescription of Ref. [18] was taken into account. A number of effective Skyrme forces can be found in literature for correct prediction of the nuclear ground-state properties. In this study, the Skyrme force SLy4 [19] has been used.

\section{$3 \quad$ Results and Discussions}

In this study, nuclear masses and experimental nuclear radii of $\sim 900$ nuclei have been used in ANNs for fitting of the charge radii. The input of the ANN was atomic masses of the nuclei and the output was nuclear radii. The whole data was partitioned into two seperated sets, $\% 80$ for the training stage and the rest for the test stage. After the training of the network, it was tested over the test set data which have never seen before by the network. As can be seen in the figure 1, the ANN predictions agree exceptionally well with experimental values.

This obviously indicates that the test set ANNs have consistently generalized the training set fittings. The MSE values were about 0.0027 and 0.0029 for the training and test stages respectively. After testing of the trained network, the whole data from ANN outputs was used for employing least square fitting. It should be noted that mass dependent form of nuclear charge radii given by the formula $R_{c}=r_{0} A^{\beta}$ has been considered for fitting. The novelly obtained formula is given by

$$
R_{c}=1.234 A^{0.28}
$$

where $R_{c}$ and $A$ is the rms charge radii and mass number of nuclei, respectively. This formula are in good agrement with the experimental data. The root mean 


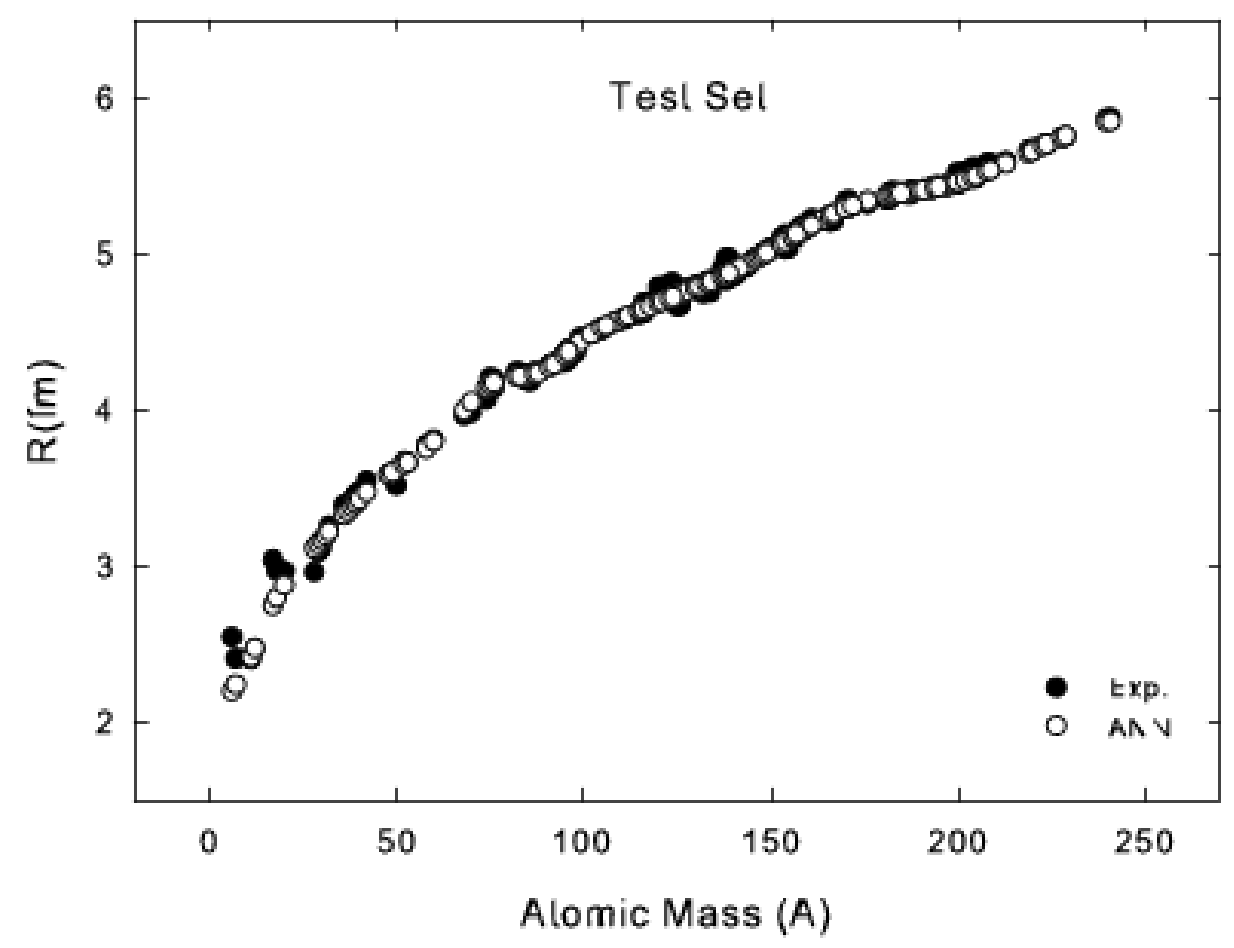

Fig. 1. The experimental (Exp.) and ANN test set predictions for nuclear radii for several isotopes ranging from $\mathrm{A}=6$ to 240 . 
square deviation between charge radii obtained from the formula and experimental ones is $\sigma=\sim 1.4 x 10^{-2}$. Beside, based on the formula we calculated the ground-state properties of nuclei such as binding energy per nucleon, two-neutron separation energy and nuclear charge radii in HFB model for Sn isotopes.
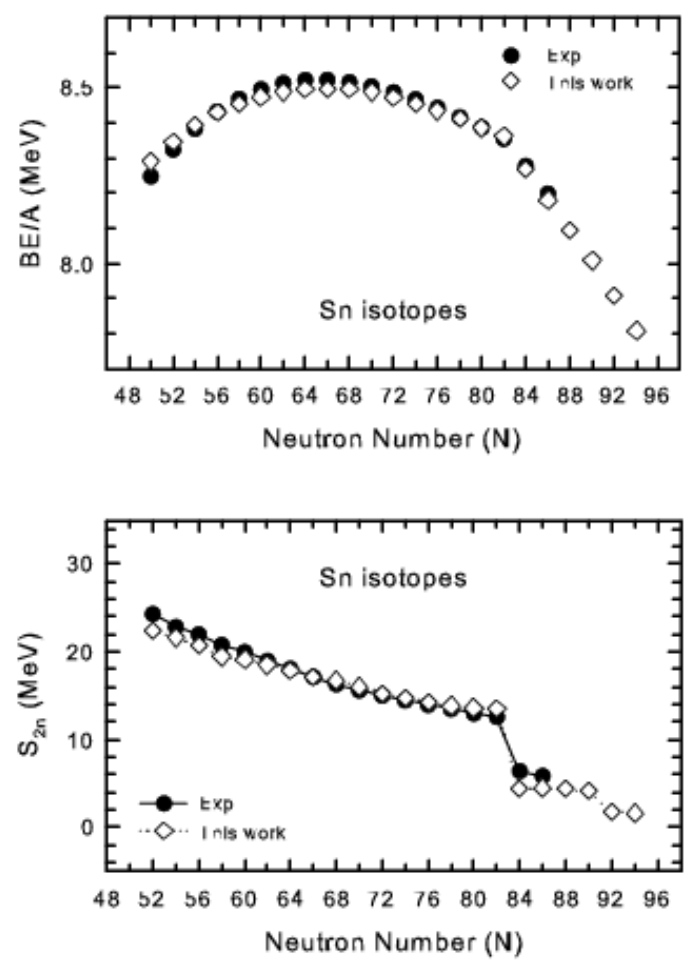

Fig. 2. Binding energies per nucleon (upper panel) and two-neutron separation energies (lower panel) for Sn Isotopes calculated with the spherical HFB code are compared with experimental data (Exp.) (from Ref. 20]).

The calculated binding energies for even-even ${ }^{100-144}$ Sn isotopes within the HFB method with the Skyrme force SLy4 are shown in the upper panel of Figure 2, Also, available experimental binding energies per nucleon taken from Ref. 20 are shown for comparison. As can be seen in the upper panel of the Figure 2, the predictions of HFB method are in good agrement with the experimental data. Maximum difference between the calculated and experimental values is only $\sim 0.04 \mathrm{MeV}$ at neutron number $N=50$. In the lower panel of the Figure 2, the calculated two-neutron separation energies of Sn isotopes and experimental ones are shown. Abrupt decrease of the two-neutron separation 
energy at neutron number $N=82$ indicates that this nuclei has a shell closure. Thus, shell effect is clearly visible in our calculations.

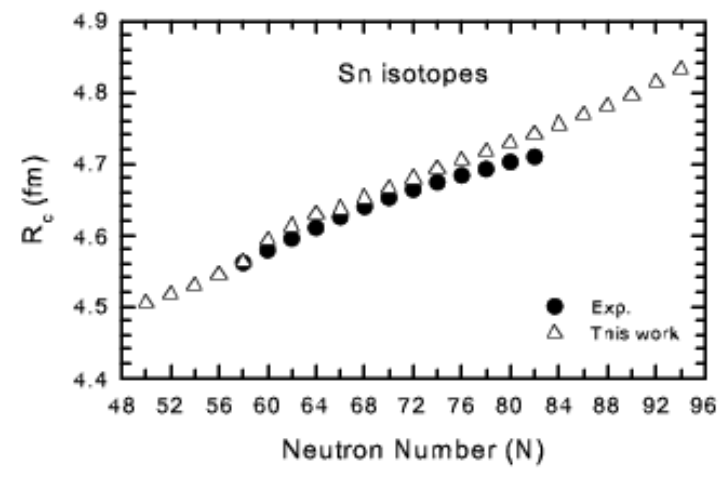

Fig. 3. The calculated rms nuclear charge radii within the HFB method with the Skyrme force SLy4 for Sn isotopes obtained by implementing the new formula in HFB code. Also, the experimental rms charge radii of Sn isotopes [4] are shown for comparison.

The calculated rms nuclear charge radii of ${ }^{100-144} \mathrm{Sn}$ in HFB method by implementing the new formula and available experimental charge radii of $\mathrm{Sn}$ isotopes taken from Ref. 4] are shown in Figure 3. As can be seen in the Figure 3 , the HFB method with the SLy4 parameters reproduced rms charge radii of Sn isotopes well. It should be noted, however, that ongoing from neutron number $N=58$ to neutron drip line, the difference between calculated and experimental charge radii for $\mathrm{Sn}$ isotopes is rising. However, maximum difference is only 0.032 fm at neutron number $N=82$.

\section{Summary}

ANNs have been applied on new experimental nuclear charge radii data to obtain simple charge radii formula based on mass dependence. The formula is founded as to be successful. By using the formula in HFB code, the ground-state properties of even-even ${ }^{100-144} \mathrm{Sn}$ isotopes such as binding energies per nucleon, twoneutron separation energies and nuclear charge radii have been calculated. The results are founded as to be successful.

\section{References}

1. Buchinger F, Crawford J E, Dutta A K, Pearson J M and Tondeur F 1994. Phys. Rev. C 49, 1402. 
2. Ring P and Schuck P, 1980 The Nuclear Many-Body Problem. Springer-Verlag, Berlin.

3. Greiner W and Maruhn J, 1996 Nuclear Models. Springer-Verlag, New York.

4. Angeli I, Gangrsky Yu P, Marinova K P, Boboshin I N, Komarov S Yu, Ishkhanov B S and Varlamov V V 2009 J. Phys. G: Nucl. Part. Phys. 36, 085102.

5. Zhang S Q, Meng J, Zhou S-G and Zeng J Y 2002 Eur. Phys. J. A 13, 285.

6. Hornik K, Stinchcombe M and White H, 1989 Neural Networks, 2, 359.

7. Athanassopoulos S, Mavrommatis E, Gernoth K A, Clark J W 2004 arXiv:nuclth/0509075

8. Athanassopoulos S, Mavrommatis E, Gernoth K A and Clark J W 2004 Nuclear Physics A 743, 222.

9. David C, Freslier M and Aichelin J 1995 Phys. Rev. C 51, 3, 1453.

10. Bass S A, Bischoff A, Maruhn J A Stöcker H and Greiner W 1996 Phys. Rev. C $53,5,2358$

11. Haddad F, Hagel K, Li J, Mdeiwayeh N, Natowitz J B, Wada R, Xiao B, David C, Freslier M and Aichelin J 1997 Phys. Rev. C 55, 3, 1371.

12. Costiris N, Mavrommatis E, Gernoth K A and Clark J W 2007 arXiv:nuclth/0701096 1 .

13. Akkoyun S, Bayram T, Kara S O and Yildiz N 2012 arXiv:nucl-th/1212.2007. (in review)

14. Haykin S, 1999 Neural Networks: A Comprehensive Foundation. Prentice-Hall Inc., Englewood Cliffs, NJ, USA.

15. Cybenko G 1989. Math. Control Signals Syst. 2, 303.

16. Levenberg K, 1944 Quart. Appl. Math., Vol. 2, 164.

17. Marquardt D, 1963. SIAM J. Appl. Math., Vol. 11, 431.

18. Stoitsov M V, Dobaczewski J Nazarewicz W and Ring P 2005 Comp. Phys. Commun. 167, 43.

19. Chabanat E, Bonche P, Haensel P, Meyer J and Schaeffer, R 1998 Nucl. Phys. A $635,231$.

20. Audi G, Wapstra A H and Thibault C 2003 Nucl. Phys. A 729, 337. 